\title{
Advanced glycation end product cross-link breaker attenuates diabetes-induced cardiac dysfunction by improving sarcoplasmic reticulum calcium handling
}

\author{
Allyson L. Kranstuber ${ }^{1,2}$, Carlos del Rio ${ }^{3}$, Brandon J. Biesiadecki ${ }^{4,5}$, Robert L. Hamlin ${ }^{3,6}$, \\ Joseph Ottobre ${ }^{2}$, Sandor Gyorke ${ }^{4,5}$ and Véronique A. Lacombe ${ }^{7 *}$ \\ ${ }^{1}$ College of Pharmacy, The Ohio State University, Columbus, OH, USA \\ ${ }^{2}$ Department of Animal Sciences, The Ohio State University College of Food, Agriculture and Environmental Sciences, Columbus, OH, USA \\ ${ }^{3}$ QTest Labs, Columbus, OH, USA \\ ${ }^{4}$ The Dorothy M. Davis Heart and Lung Research Institute, The Ohio State University, Columbus, OH, USA \\ ${ }^{5}$ Department of Physiology and Cell Biology, The Ohio State University College of Medicine, Columbus, OH, USA \\ ${ }^{6}$ College of Veterinary Medicine, The Ohio State University, Columbus, OH, USA \\ 7 Department of Physiological Sciences, Oklahoma State University, Stillwater, OK, USA
}

\section{Edited by:}

Jean-Pierre Montani, University of Fribourg, Switzerland

Reviewed by:

Lea M. D. Delbridge, University of Melbourne, Australia

Candice M. Thomas, Texas A\&M, USA

Peter Celec, Comenius University, Slovakia

Yan Guo, The First Affiliated Hospital of Nanjing Medical University, China

*Correspondence:

Véronique A. Lacombe, Department of Physiological Sciences, Oklahoma State University, 283 McElroy Hall,

Stillwater, OK 74078, USA.

e-mail:veronique.lacombe@

okstate.edu
Diabetic heart disease is a distinct clinical entity that can progress to heart failure and sudden death. However, the mechanisms responsible for the alterations in excitation-contraction coupling leading to cardiac dysfunction during diabetes are not well known. Hyperglycemia, the landmark of diabetes, leads to the formation of advanced glycation end products (AGEs) on long-lived proteins, including sarcoplasmic reticulum (SR) $\mathrm{Ca}^{2+}$ regulatory proteins. However, their pathogenic role on $\mathrm{SR} \mathrm{Ca}^{2+}$ handling in cardiac myocytes is unknown. Therefore, we investigated whether an AGE cross-link breaker could prevent the alterations in $\mathrm{SR} \mathrm{Ca}^{2+}$ cycling that lead to in vivo cardiac dysfunction during diabetes. Streptozotocin-induced diabetic rats were treated with alagebrium chloride (ALT-711) for 8 weeks and compared to age-matched placebo-treated diabetic rats and healthy rats. Cardiac function was assessed by echocardiographic examination. Ventricular myocytes were isolated to assess SR $\mathrm{Ca}^{2+}$ cycling by confocal imaging and quantitative Western blots. Diabetes resulted in in vivo cardiac dysfunction and ALT-711 therapy partially alleviated diastolic dysfunction by decreasing isovolumetric relaxation time and myocardial performance index (MPI) (by 27 and $41 \%$ vs. untreated diabetic rats, respectively, $P<0.05)$. In cardiac myocytes, diabetes-induced prolongation of cytosolic $\mathrm{Ca}^{2+}$ transient clearance by $43 \%$ and decreased SR $\mathrm{Ca}^{2+}$ load by $25 \%$ $(P<0.05)$; these parameters were partially improved after ALT-711 therapy. SERCA2a and RyR2 protein expression was significantly decreased in the myocardium of untreated diabetic rats (by 64 and $36 \%$ vs. controls, respectively, $P<0.05$ ), but preserved in the treated diabetic group compared to controls. Collectively, our results suggest that, in a model of type 1 diabetes, AGE accumulation primarily impairs SR $\mathrm{Ca}^{2+}$ reuptake in cardiac myocytes and that long-term treatment with an AGE cross-link breaker partially normalized $\mathrm{SR} \mathrm{Ca}^{2+}$ handling and improved diabetic cardiomyopathy.

Keywords: cardiomyopathy, sarcoplasmic reticulum $\mathrm{Ca}^{2+}$-ATPase pump, diastolic function, type 1 diabetes, alagebrium chloride (ALT-711)

\section{INTRODUCTION}

Diabetes has become an epidemic disease and it is estimated that by the year 2025, it will affect over 300 million people worldwide (Amos et al., 1997; Boudina and Abel, 2007). In the United States alone, about $8 \%$ of the population is affected by diabetes and approximately one million of those people suffer from insulindependent (type 1) diabetes. Type 1 diabetes is characterized by sustained hyperglycemia resulting from the loss of insulinproducing pancreatic beta cells. This loss in insulin production results in dysfunctional glucose uptake in insulin-sensitive tissues (e.g., striated muscle) and causes multiple-organ complications. Of importance, diabetes is also a common cause of cardiovascular diseases. Within the past 30 years, diabetic cardiomyopathy has been identified as its own clinical unit, independent of coronary artery disease and atherosclerosis (Fang et al., 2004; Poornima et al., 2006). Ventricular diastolic dysfunction is the first stage of diabetic cardiomyopathy and has been reported in about $50 \%$ of asymptomatic patients (Fang et al., 2004; Lacombe et al., 2007). Because intracellular calcium $\left(\mathrm{Ca}^{2+}\right)$ homeostasis is crucial for excitation-contraction coupling, chronic diabetes mellitus has been associated with impaired cardiac contractility and relaxation of the myocardium due to altered $\mathrm{Ca}^{2+}$ homeostasis (LagadicGossmann et al., 1996; Pierce and Russell, 1997; Netticadan et al., 2001; Zhong et al., 2001; Choi et al., 2002; Fang et al., 2004; 
Lacombe et al., 2007). However, the exact mechanisms for this impaired $\mathrm{Ca}^{2+}$ homeostasis and the specific therapeutic strategies for this patient population remain elusive.

The sarcoplasmic reticulum (SR) functions as the main regulator of intracellular $\mathrm{Ca}^{2+}$ and is a major determinant of cardiac contraction and relaxation (Bers, 2002). $\mathrm{Ca}^{2+}$ entry through the L-type $\mathrm{Ca}^{2+}$ channel activates $\mathrm{Ca}^{2+}$ release from the SR. The SR $\mathrm{Ca}^{2+}$ release channels, the ryanodine receptors (RyRs), release the majority of free $\mathrm{Ca}^{2+}$ necessary for contraction, and $\mathrm{SR} \mathrm{Ca}^{2+}$ ATPase (SERCA2a) pumps sequester the majority of $\mathrm{Ca}^{2+}$ during relaxation of cardiac myocytes. Several groups (including ours) have reported decreased expression of these SR $\mathrm{Ca}^{2+}$ regulatory proteins in type 1 diabetic rats with cardiac dysfunction (Poornima et al., 2006; Lacombe et al., 2007; Ratnadeep et al., 2009). Furthermore, impaired excitation-contraction coupling in diabetic myocytes has been characterized by slower $\mathrm{Ca}^{2+}$ transient decays and cytosolic $\mathrm{Ca}^{2+}$ overload during the diastolic phase (Pierce and Russell, 1997; Choi et al., 2002; Lacombe et al., 2007). However, the mechanisms by which SR $\mathrm{Ca}^{2+}$ cycling is impaired during diabetic cardiomyopathy have not been fully elucidated.

Chronic hyperglycemia, the hallmark of diabetes, accelerates the reaction between glucose and proteins and leads to the formation of advanced glycation end products (AGEs). These AGEs form irreversible cross-links throughout the lifetime of many large proteins (such as collagen and hemoglobin), covalently modifying their structure and function (Cooper, 2004). Therefore, AGEs induce myocardial fibrosis and stiffness leading to severe cardiac dysfunction (Norton et al., 1996; Asif et al., 2000; Vaitkevicius et al., 2001; Aronson, 2003; Candido et al., 2003; Bakris et al., 2004; Cooper, 2004; Hartog et al., 2007; Ma et al., 2009). In addition, Bidasee et al. $(2003,2004)$ have demonstrated the presence of cross-linked AGEs on long-lived intracellular cardiac SR proteins such as the SERCA2a pump and RyR2 after a few weeks of diabetes. Therefore, one could hypothesize that the post-translational modifications of the SR proteins by AGEs could lead to an alteration in $\mathrm{Ca}^{2+}$ homeostasis. However, the functional significance of AGEs on $\mathrm{SR} \mathrm{Ca}^{2+}$ regulatory proteins in cardiac myocytes and thus on excitation-contraction coupling has not been determined. Our hypothesis was that treatment with an antiglycation therapeutic agent, dimethyl-3-phenacylthiazolium chloride (alagebrium chloride or ALT-711), which chemically breaks AGE cross-links, will normalize SR $\mathrm{Ca}^{2+}$ reuptake in cardiac myocytes and therefore improve diastolic function in type 1 diabetes.

\section{MATERIALS AND METHODS ANIMAL MODEL}

Eight-week-old male Wistar rats were randomly divided into 3 groups ( $n=11$ /group): untreated age-matched control group (CON); untreated diabetic group (DX); and ALT-711 (Shanghai Inc., China) treated diabetic group (DX-ALT). Diabetes was induced at 10 weeks of age in DX and DX-ALT groups by a single injection of streptozotocin (STZ, $50 \mathrm{mg} / \mathrm{kg}$ IP diluted in $1 \mathrm{~mL}$ citrate buffer). The control group received similar volume of vehicle. One diabetic group received dimethyl-3phenacylthiazolium chloride (ALT-711, $10 \mathrm{mg} / \mathrm{kg}$ per day in the drinking water) for 8 weeks. This therapeutic dose has been previously shown to significantly reduce cardiac AGE level in STZ-induced diabetes (Candido et al., 2003). The volume of ALT-711 delivered in the drinking water was calculated based on the individual water consumption, which was measured every other day. To confirm the status of diabetes, venous blood samples were drawn from the tail vein for measurement of blood glucose concentration using a glucometer (BD Logic) at baseline and then weekly after STZ injection for 8 weeks. Animals were weighed once a week, as a means to monitor their clinical condition. This animal protocol was approved by the Ohio State University Institutional Animal Care and Use Committee.

\section{ECHOCARDIOGRAPHY}

Transthoracic echocardiographic examination was performed to assess systolic and diastolic function at baseline and 8 weeks after the induction of diabetes. Two-dimensional, M-mode, and pulsed-wave Doppler imaging were obtained in rats lightly anesthetized with isoflurane (minimal effective concentration), and placed on a heating table to maintain normothermia. Examinations were done using a high-resolution high-frequency digital imaging system with a $21 \mathrm{MHz}$ linear-array transducer and simultaneous ECG recording (Vevo 2100, VisualSonics, Toronto, Canada), following standard techniques as previously described (Dirksen et al., 2007; Lacombe et al., 2007, 2010; Ware et al., 2011). Standard parasternal long- and short-axis views (6-8/rat) were obtained during each echocardiographic examination. Ventricular structure and function were assessed by twodimensional cine loops of a long-axis view (with frame rates of at least 200 frames/s) and of a short-axis view at mid-level of the papillary muscles, as well as M-mode loops of the short-axis view. Thicknesses of the interventricular septum and of the left ventricular posterior wall, and left ventricular internal diameter (LVID) were measured in systole and diastole from the short-axis view according to standard procedures. Left ventricular (LV) ejection fraction (EF), a surrogate of systolic function, was calculated, as follows: $\mathrm{EF}=$ (LVID end-diastolic - LVID end-systolic/LVID enddiastolic) $\times 100 \%$. The apical four-chamber view was used for color flow guided, pulsed-wave Doppler imaging of transmitral flow and LV outflow. The myocardial performance index (MPI or TEI index) was obtained from the sum of the LV isovolumic relaxation time and isovolumic contraction time divided by the aortic ejection time, parameters which were measured from the pulsed-wave Doppler imaging of transmitral flow and LV outflow. Echocardiographic image measurements were performed offline. All image acquisitions and offline measurements were conducted by the same investigator (AK). Average values were obtained from the measurement of three cardiac cycles from one cine loop.

\section{MASSON TRICHROME STAINING}

LV fibrosis was measured at 8 weeks after the induction of diabetes by the Ohio State University's Core Pathology laboratory. LV cross sections were washed with PBS, fixed using OCT (optimal cutting temperature) compound, frozen in dry ice and stained with Masson Trichrome staining. 


\section{VENTRICULAR MYOCYTE ISOLATION}

Following echocardiographic measurements, animals were euthanized by pentobarbital sodium. The heart was removed and perfused in a retrograded manner, using a Langendorff apparatus with tyrode buffer $\left(37^{\circ} \mathrm{C}, \mathrm{pH}=7.35\right.$ and oxygenated with $95 \%$ $\mathrm{O}_{2}$ and $5 \% \mathrm{CO}_{2}$ ), which contained (in $\mathrm{mM}$ ): $\mathrm{NaCl}(135), \mathrm{KCl}$ (5.4), $\mathrm{MgCl}_{2}$ (1), $\mathrm{NaH}_{2} \mathrm{PO}_{4}$ (0.33), Hepes (10), glucose (10), and $\mathrm{CaCl}_{2}$ (1). This initial perfusion was followed by a perfusion with tyrode buffer without any $\mathrm{CaCl}_{2}$. Subsequently, collagenase (type II, Worthington Biochemical, $1 \mathrm{mg} / \mathrm{ml}$ ) was added to the calcium free tyrode buffer and recirculated for the rest of the perfusion period. When the heart was soft, the ventricles were minced and the cells were subsequently washed in tyrode solution containing $\mathrm{CaCl}_{2}$ (1). Only rod-shaped cells with sharp margins and clear striations were included in the study. All recordings were made within $5 \mathrm{~h}$ of isolation (Dirksen et al., 2007; Lacombe et al., 2007, 2010).

\section{MEASUREMENT OF $\mathrm{Ca}^{2+}$ TRANSIENT AND SR $\mathrm{Ca}^{2+}$ LOAD}

$\mathrm{Ca}^{2+}$ transient was measured in fluo-3-loaded cardiac myocytes with confocal $\mathrm{Ca}^{2+}$ imaging as previously described; for measurements of $\mathrm{Ca}^{2+}$ transients and transient decay, mean area under the curve was calculated (Kubalova et al., 2005; Dirksen et al., 2007; Lacombe et al., 2007, 2010). Rapid applications of caffeine $(10 \mathrm{mM})$ were used to measure SR $\mathrm{Ca}^{2+}$ content by measuring the peak amplitude of the caffeine-induced $\mathrm{Ca}^{2+}$ transients. Intracellular $\mathrm{Ca}^{2+}$ imaging was performed using a Laser Scanning Confocal System (Olympus Fluoview 1000 confocal microscope interfaced to an IX-70 inverted microscope and equipped with an $60 \times 1.4$ NA oil objective). Fluo-3 was excited by the $488-\mathrm{nm}$ beam of an argon-ion laser, and the fluorescence was acquired at wavelengths $>515 \mathrm{~nm}$ in the line scan mode, at a rate of 2 or $6 \mathrm{~ms}$ per scan. The magnitude of fluorescent signals was quantified in terms of F/F0, where F0 is baseline fluorescence (Kubalova et al., 2005; Dirksen et al., 2007; Lacombe et al., 2007, 2010).

\section{MEASUREMENTS OF SR $\mathrm{Ca}^{2+}$ REGULATORY PROTEINS}

LV myocardium was collected 8 weeks after the induction of diabetes. Crude membrane homogenates were prepared for Western blot analysis, as previously described (Meurs et al., 2006; Lacombe et al., 2007; Ware et al., 2011). Proteins were subjected to sodium dodecyl sulphate-polyacrylamide gel electrophoresis (SDS-PAGE) electrophoresis, electrophoretically transferred to PVDF membranes using a trans-blot cell (BioRad Laboratories, Hercules, CA, USA; Meurs et al., 2006; Lacombe et al., 2007; Ware et al., 2011). Samples from the 3 groups were loaded on the same gel to ensure equal blotting conditions for each group. Membrane proteins were incubated with mouse RyR2 or SERCA2a antibodies (1:3000 and 1:1000 dilution, respectively, Affinity Bioreagents), and subsequently with the appropriate secondary antibodies conjugated to horseradish peroxidase (1:50,000 dilution, Jackson ImmunoResearch Laboratories; 1:5000 dilution, Sigma Aldrich, respectively). Quantitative determination of protein was performed by autoradiography after revealing the antibody-bound protein by enhanced chemiluminescence reaction. The data were normalized to actin or calsequestrin, previously quantified by reprobing each membrane with calsequestrin polyclonal IgG (Calbiochem) or Actin monoclonal IgG (Sigma Aldrich), respectively.

\section{STATISTICAL ANALYSIS}

A Two-Way ANOVA (treatment and time factors) for the in vivo measurements, and a one-way analysis of variance (treatment factor) for the in vitro measurements were performed, as appropriate. Data were reported as means \pm SE. Statistical significance was defined as $P<0.05$.

\section{RESULTS}

As expected, the STZ-treated rats exhibited hyperglycemia within $72 \mathrm{~h}$ post injection, which persisted during the 8-week experimental period $(P<0.05$, Figure 1). Treatment with an AGE cross-link breaker, ALT-711, for 8 weeks did not significantly alter blood glucose concentration compared with untreated diabetic rats. In addition, the diabetic rats had a significantly lower body weight when compared to the control group and there was a tendency $(P<0.1)$ for the treated diabetic rats to have a higher body weight than the untreated diabetic rats at 4 and 8 weeks after the induction of diabetes (Figure 2).

\section{In vivo VENTRICULAR FUNCTION}

We then evaluated the effect of diabetes and ALT-711 therapy on systolic and diastolic function by echocardiographic examination in (treated and untreated) diabetic and control groups. EF, a surrogate of systolic function, was mildly decreased at 8 weeks after the induction of diabetes compared to baseline values (Table 1). In addition, the isovolumic relaxation time and the MPI, two parameters of LV relaxation, were significantly increased in untreated diabetic rats compared to the age-matched control groups (Table 2). ALT-711 therapy did not significantly alter EF of the diabetic myocardium but blunted the increase in isovolumic relaxation time and the MPI in diabetic animals, suggesting

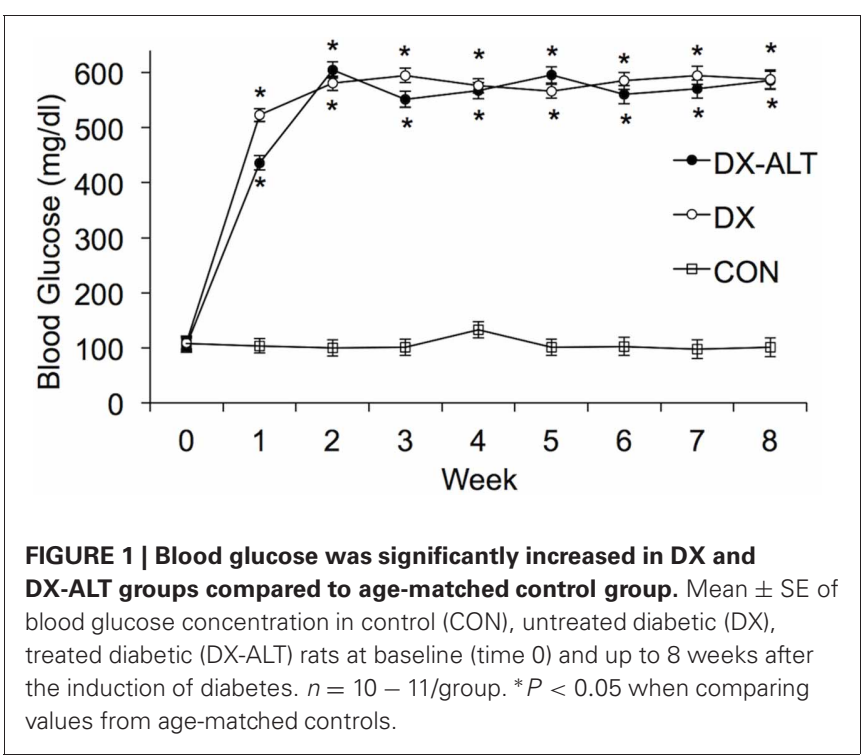




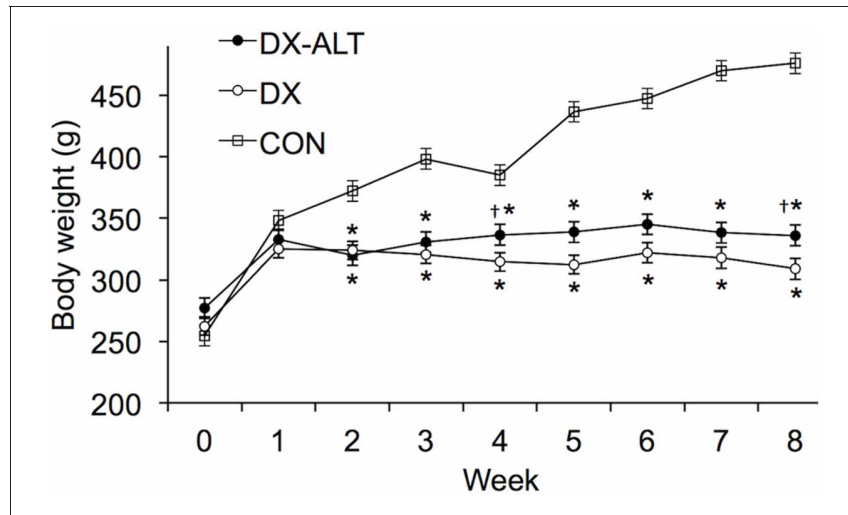

FIGURE 2 | Body weight was significantly decreased in DX and DX-ALT groups compared to age-matched control group. Mean \pm SE of body weight in control (CON), untreated diabetic (DX), treated diabetic (DX-ALT) rats at baseline (time 0 ) and 8 weeks after the induction of diabetes. $n=10-11$ /group. $* P<0.05$ when comparing values from age-matched controls. ${ }^{\dagger} P<0.1$ when comparing values from $\mathrm{DX}$ group. that ALT-711 therapy partially prevented cardiac dysfunction by primarily improving diastolic dysfunction (Figure $\mathbf{3}$ and Table 2).

Because diastolic dysfunction may be due to reduced rate of sequestration of $\mathrm{Ca}^{2+}$ into the SR or to histological changes in myocardium rendering it less compliant, hearts were stained with Masson's trichrome to quantify fibrosis. Masson trichrome staining did not reveal significant fibrosis of the left ventricle in (treated and untreated) diabetic rats compared with the control group, suggesting primarily an impaired ventricular relaxation rather than increased myocardial stiffness in our diabetic model (Figure 4).

\section{MEASUREMENT OF Ca ${ }^{2+}$ TRANSIENT AND SR $\mathrm{Ca}^{2+}$ LOAD}

Because AGEs accumulate on SR $\mathrm{Ca}^{2+}$ regulatory proteins and could alter their function leading to diabetic cardiomyopathy, we further determined if treatment with an AGE cross-link breaker would improve SR $\mathrm{Ca}^{2+}$ handling during diabetic cardiomyopathy by measuring $\mathrm{Ca}^{2+}$ transient in fluo-3-loaded cardiac myocytes with confocal $\mathrm{Ca}^{2+}$ imaging. There was a significant

Table 1 | Parameters derived from M-mode echocardiography of LV for age-matched control (CON), untreated diabetic (DX), treated diabetic (DX-ALT) groups at baseline and at 8 weeks after the induction of diabetes.

\begin{tabular}{|c|c|c|c|c|c|c|}
\hline & \multicolumn{2}{|c|}{ CON } & \multicolumn{2}{|c|}{ DX } & \multicolumn{2}{|c|}{ DX-ALT } \\
\hline EF (\%) & $85.7 \pm 2.6$ & $84.4 \pm 1.8$ & $86.11 \pm 2.02$ & $67.8 \pm 2.54^{* \#}$ & $83.7 \pm 2.08$ & $73.8 \pm 0.14^{* \#}$ \\
\hline FS (\%) & $57.7 \pm 3.11$ & $55.7 \pm 2.22$ & $57.7 \pm 2.50$ & $39.6 \pm 2.07^{* \#}$ & $54.4 \pm 2.42$ & $44.5 \pm 1.71^{* \#}$ \\
\hline IVS;d (cm) & $1.85 \pm 0.07$ & $2.11 \pm 0.07$ & $2.05 \pm 0.07$ & $1.85 \pm 0.10$ & $2.02 \pm 0.10$ & $1.84 \pm 0.09$ \\
\hline IVS;s (cm) & $3.42 \pm 0.07$ & $3.76 \pm 0.14^{\#}$ & $3.64 \pm 0.11$ & $2.94 \pm 0.09^{* \#}$ & $3.54 \pm 0.10$ & $3.15 \pm 0.09^{* \#}$ \\
\hline LVPW;d (cm) & $1.64 \pm 0.07$ & $1.84 \pm 0.09$ & $1.70 \pm 0.08$ & $1.63 \pm 0.10$ & $1.63 \pm 0.08$ & $1.60 \pm 0.08$ \\
\hline LVPW;s (cm) & $3.17 \pm 0.12$ & $3.43 \pm 0.1^{\#}$ & $3.11 \pm 0.12$ & $2.57 \pm 0.08^{* \#}$ & $2.95 \pm 0.12$ & $2.67 \pm 0.14^{*}$ \\
\hline LV Mass;c (mg) & $610.1 \pm 42.5$ & $891.3 \pm 36.2^{\#}$ & $676.8 \pm 35.8$ & $812.9 \pm 70.4^{\#}$ & $663.2 \pm 35.3$ & $792.6 \pm 41.2$ \\
\hline LV Vol;d (ul) & $216.5 \pm 16.5$ & $289.6 \pm 11.3^{\#}$ & $215.9 \pm 11.45$ & $322.1 \pm 15.9^{\#}$ & $226.6 \pm 19.6$ & $323.2 \pm 18.4^{\#}$ \\
\hline $\mathrm{HR}$ (b/min) & $329.6 \pm 9.21$ & $318.24 \pm 8.64$ & $344.7 \pm 2.00$ & $243.8 \pm 7.95^{* \#}$ & $335.4 \pm 10.5$ & $268.3 \pm 6.02^{* \#}$ \\
\hline
\end{tabular}

Data are mean \pm SE for $n=9-11 /$ group. IVS; s: interventricular septal dimension-systole, IVS; $d$ : interventricular septal dimension-diastole, LVPW; : left ventricular posterior wall dimension-systole, LVPW; d: left ventricular posterior wall dimension-diastole, LVID;s: left ventricular internal diameter-systole, LVID; $d$ : left ventricular internal diameter-diastole; LV Mass;c: left ventricular mass corrected, EF: ejection fraction, FS: fractional shortening, HR: heart rate. ${ }^{*} P<0.05$ when comparing values from age-matched controls for same time point. ${ }^{\#} P<0.05$ when comparing values from baseline.

Table 2 | Doppler-derived parameters of diastolic function for age-matched control (CON), untreated diabetic (DX), treated diabetic (DX-ALT) groups at baseline and at 8 weeks after the induction of diabetes.

\begin{tabular}{lllllll}
\hline & \multicolumn{2}{c}{ CON } & \multicolumn{2}{c}{ DX } & \multicolumn{2}{c}{ DX-ALT } \\
\cline { 2 - 7 } & Baseline & $\mathbf{8}$ weeks & Baseline & $\mathbf{8}$ weeks & Baseline & $\mathbf{8}$ weeks \\
\hline MV E/A & $1.52 \pm 0.06$ & $1.52 \pm 0.06$ & $1.62 \pm 0.092$ & $1.64 \pm 0.080$ & $1.59 \pm 0.11$ & $1.82 \pm 0.15$ \\
IVRT & $22.4 \pm 0.83$ & $25.4 \pm 1.07$ & $23.0 \pm 0.95$ & $44.98 \pm 1.88^{* * \#}$ & $24.8 \pm 1.14$ & $37.3 \pm 2.07^{* \dagger}$ \\
MV Dec Acc $\left(\mathrm{mm} / \mathrm{s}^{2}\right)$ & $38647.0 \pm 4166.0$ & $34047.0 \pm 4493.3$ & $39465.7 \pm 3418.6$ & $39593.8 \pm 8829.9$ & $40454.0 \pm 4650.1$ & $26790.0 \pm 2305.4$ \\
LV MPI & $0.54 \pm 0.04$ & $0.57 \pm 0.05$ & $0.46 \pm 0.03$ & $0.76 \pm 0.08^{* \#}$ & $0.61 \pm 0.04$ & $0.56 \pm 0.08^{\dagger}$ \\
\end{tabular}

Data are mean $\pm S E$ for $n=9-11 /$ group. MV E, maximum velocity of the $E$ wave; $M V A$, maximum velocity of the $A$ wave; $M V E / A$, ratio of $E$ and $A$ wave; IVRT, isovolumic relaxation time; MV Dec Acc, maximum velocity deceleration acceleration; $L V M P l$, left ventricle myocardial performance index. ${ }^{*} P<0.05$ when comparing values from age-matched controls for same time point. ${ }^{\#} P<0.05$ when comparing values from baseline. ${ }^{\dagger} P<0.05$ when comparing DX values vs. DX-ALT. 


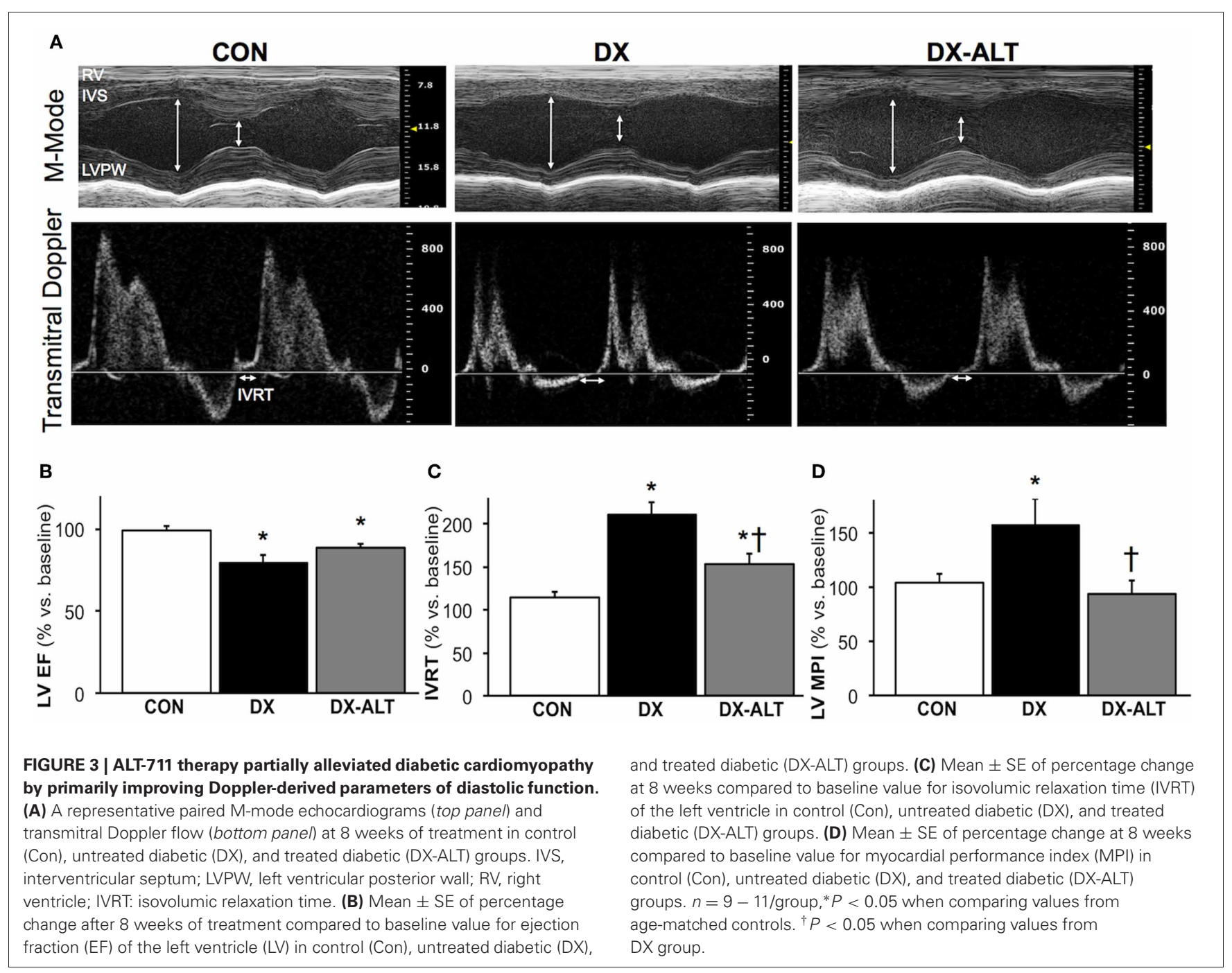

$(P<0.001)$ decrease in $\mathrm{Ca}^{2+}$ transient amplitude in isolated cardiac myocytes of (untreated and treated) diabetic rats when compared with controls (Figure 5). In addition, the $\mathrm{Ca}^{2+}$ transient decay during the diastolic phase was significantly prolonged in diabetic compared with control myocytes (Figure 5). ALT-711 therapy resulted in shortening of the $\mathrm{Ca}^{2+}$ transient decay, suggesting an improvement in $\mathrm{SR} \mathrm{Ca}^{2+}$ reuptake in treated diabetic myocytes. In addition, SR $\mathrm{Ca}^{2+}$ load, measured by caffeineevoked $\mathrm{Ca}^{2+}$ transient amplitudes, was significantly decreased in myocytes from untreated diabetic, but not from treated diabetic rats, compared with control groups (Figure 5), suggesting that ALT-711 therapy partially attenuated SR $\mathrm{Ca}^{2+}$ content depletion in diabetic myocytes.

\section{MEASUREMENT OF SR $\mathrm{Ca}^{2+}$ REGULATORY PROTEINS}

To determine if AGE accumulation could alter the expression of SR $\mathrm{Ca}^{2+}$ regulatory protein, we performed quantitative immunoblot analysis of SERCA2a and RyR2. SERCA2a pump expression was significantly decreased in the myocardium of untreated diabetic, but not from treated diabetic rats, compared with control groups. We also observed a decrease in RyR2 protein expression in the myocardium of diabetic rats when compared to controls, while the diabetic treated group exhibited similar RyR2 protein levels compared with controls (Figure 6).

\section{DISCUSSION}

The major finding of this study was that long-term treatment with ALT-711, an AGE cross-link breaker, partially restored SR $\mathrm{Ca}^{2+}$ handling in cardiac myocytes, by primarily improving $\mathrm{Ca}^{2+}$ transient decay compared to the untreated diabetic rats. As a result, ALT-711 therapy partially prevented in vivo diastolic dysfunction in the diabetic myocardium of a rodent model of type 1 diabetes.

The STZ diabetic rat model is a well-established model to study insulin-dependent (type 1) diabetes. STZ contains a glucose molecule with a highly reactive nitrosourea side chain, which initiates a specific cytotoxic action on the pancreatic $\beta$-cell. A few weeks after STZ injection, rodents develop biochemical and functional myocardial abnormalities, which are the result of chronic hyperglycemia rather than a direct effect of the drug 

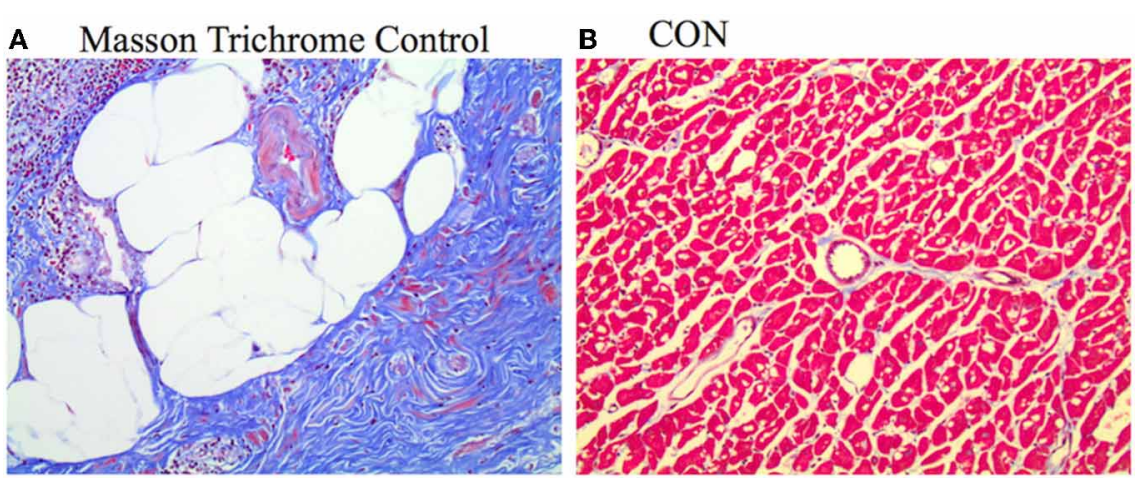

c DX

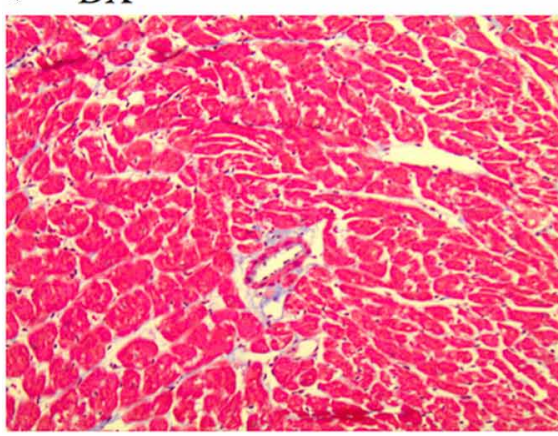

FIGURE 4 | Lack of fibrosis in ventricle of (untreated and treated) diabetic rats. (A) Masson Trichrome staining showing fibrosis (Fibrosis Control experiment). (B-D) Representative Trichrome staining ( $\times 20$ objective)

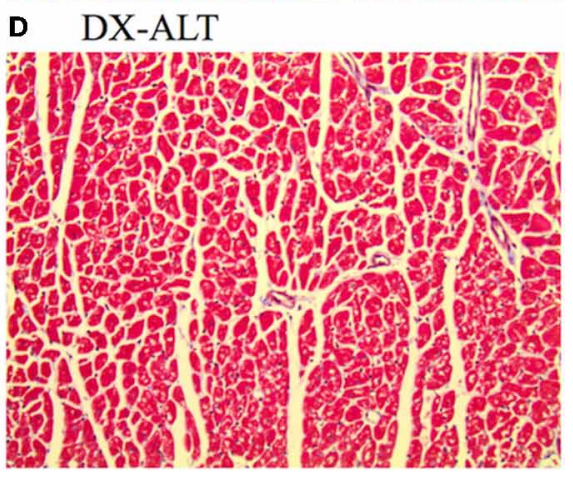

demonstrating the lack of ventricle fibrosis in age-matched control (Con), untreated diabetic (DX), and treated diabetic (DX-ALT) groups. itself. Therefore, diabetic rodents display clinical signs (hyperglycemia, polydipsia, glycosuria, and polyuria) and cardiovascular complications similar to those in human diabetic patients. Since a close relationship between the STZ dose and the severity of diabetes has been demonstrated and since other parameters (such as animal strain, frequency or route of injection, or preparation of STZ, and duration of diabetes) all significantly influence the severity of the model, we previously established in our laboratory a protocol (using a low dose of STZ) to induce a mild form of diabetes and to mimic the early metabolic and cardiac events that occur in diabetic subjects (Lacombe et al., 2007). As a result, the mortality rate was less than $5 \%$ and all the rats became diabetic. Importantly, these diabetic rats develop primarily mild diastolic dysfunction followed by mild systolic dysfunction, have prolonged QTs and action potential durations and are prone to arrhythmias (Lacombe et al., 2007, 2010). In addition, this animal model is somewhat relevant to non-insulin-dependent diabetic (or type 2) subjects, who also develop diabetic cardiomyopathy (Boudina and Abel, 2007). Indeed, while initially there is insulin resistance in type 2 diabetes, as the disease progresses there is also insulin deficiency secondary to the exhaustion of pancreatic beta cells (which have produced large amounts of insulin to compensate for the insulin resistance).

Diabetic heart disease, also referred to as diabetic cardiomyopathy, is a major cause of cardiovascular diseases in the United States today. It can lead to heart failure and sudden death, killing $\sim 65 \%$ of the patient population (Choi et al., 2002). The presence of LV diastolic dysfunction is an early complication of diabetes and is the first stage in the development of diabetic cardiomyopathy (Fang et al., 2004; Lacombe et al., 2007). Diastolic dysfunction refers to mechanical and functional abnormalities such as impairment of diastolic distensibility, filling, or relaxation of the left ventricle (Aurigemma et al., 2006). The incidence of diastolic dysfunction has been underestimated until the recent advancement of non-invasive imaging tools of cardiac relaxation, such as Doppler flow and tissue Doppler imaging. In particular, the MPI is a Doppler-derived parameter independent of blood pressure and load. MPI increases with worsening of LV diastolic dysfunction, even during the early stages of subclinical diastolic dysfunction (Su et al., 2006). Early determination of this myocardial manifestation of diabetes is of major importance, since subclinical diastolic dysfunction contributes to a four to eightfold increase in risk for congestive heart failure in diabetic patients (Piccini et al., 2004). As previously reported by our group (Lacombe et al., 2007, 2010), this animal model displayed mild diastolic dysfunction, as evident by the alterations of Doppler flow-derived parameters (i.e., increased isovolumic relaxation time and MPI). Since early relaxation is an active process regulated by SR $\mathrm{Ca}^{2+}$ handling, impaired myocardial relaxation is characterized by disturbances in calcium homeostasis rather than by fibrosis (Fang et al., 2004; Lacombe et al., 2007). Similarly, we did not detect significant amount of fibrosis in (untreated and treated) diabetic rats, suggesting that the impaired ventricular relaxation rather than increased myocardial stiffness primarily accounts for the 


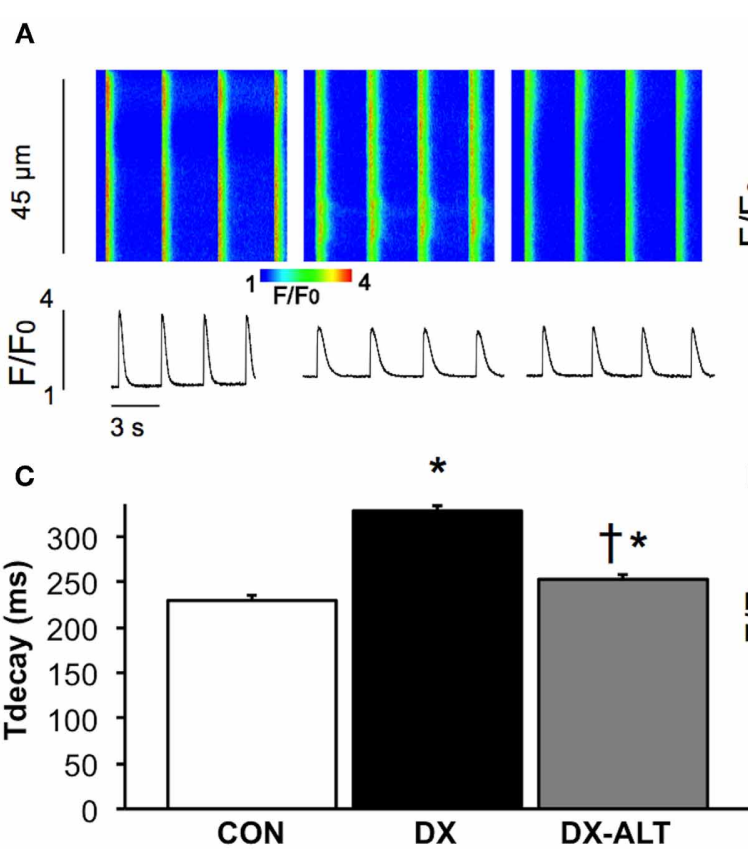

FIGURE 5 | ALT-711 therapy ablated the prolongation of $\mathrm{Ca}^{2+}$ transient decay in diabetic cardiac myocytes. (A) Representative confocal line scan images of $\mathrm{Ca}^{2+}$ transient along with their spatial averages in myocytes from age-matched control (CON, left), untreated diabetic (DX, middle), and treated diabetic (DX-ALT, right) rats. F0, diastolic fluorescence. (B) Mean $\pm \mathrm{SE}$ of $\mathrm{Ca}^{2+}$ transient amplitude (F/FO) for $\mathrm{CON}$, DX, and DX-ALT rats, $n=43-44$ /group. (C) Mean \pm SE of the time constant $(\tau)$ of $\mathrm{Ca}^{2+}$ transient decay in CON, DX, and DX-ALT rats. $n=40 \pm 4$ /group.

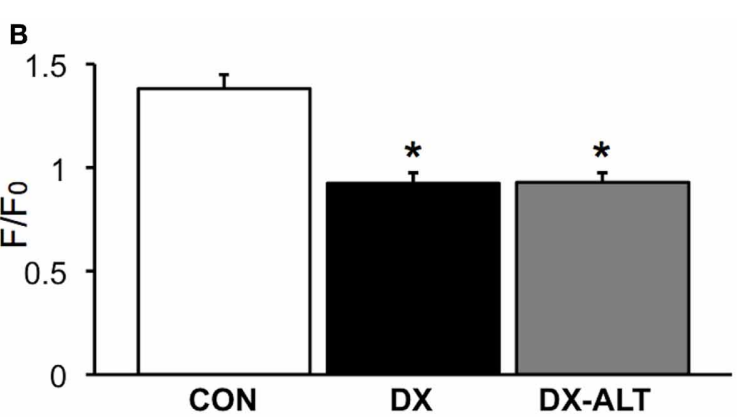

D

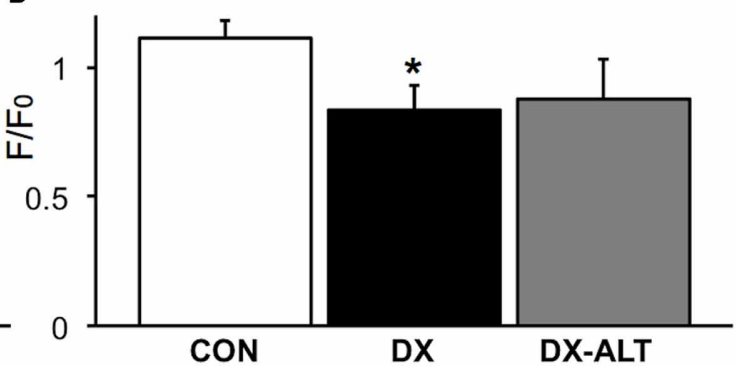

$\tau$ decay is significantly increased in DX compared to CON $(P<0.05)$. Please note a significant decrease in DX-ALT compared to DX, showing an improvement in calcium reuptake time after 8 weeks of ALT-711 treatment.

(D) Caffeine-induced $\mathrm{Ca}^{2+}$ transient amplitudes (mean $\pm \mathrm{SE}$ ) were reduced in myocytes from diabetic compared with control and treated diabetic rats. $n=4-5 /$ group, $* P<0.05$ when comparing values from age-matched control myocytes. ${ }^{\dagger} P<0.05$ when comparing values from $\mathrm{DX}$ group. negative lusitrope manifested during ventricular filling in our type 1 diabetic model. This is in agreement with previous studies that reported no difference in myocardial collagen (vs. control group) in a similar type 1 diabetic model exhibiting mild diastolic dysfunction (Dent et al., 2001). In addition, it has been suggested that diabetes mellitus can produce diastolic dysfunction before the development of myocardial fibrosis due to formation of AGEs, although the mechanisms were not investigated (Norton et al., 1996; Fang et al., 2004). Therefore, this model of mild diastolic dysfunction allows us to evaluate the effect of AGEs on ventricular relaxation and its underlying alterations in $\mathrm{SR} \mathrm{Ca}^{2+}$ homeostasis before the development of marked fibrosis.

AGEs are proteins that accumulate in the plasma of diabetic patients as a result of the persistent hyperglycemia and are closely linked with cardiovascular diseases. During diabetes, and to a lesser extent during aging, AGEs also accumulate at an accelerated rate in various cell types (in days to weeks) and produce multipleorgan dysfunction (Cooper, 2004; Hartog et al., 2007). In the heart, AGE accumulation contributes to diastolic dysfunction, by inducing myocardial fibrosis and stiffness (Norton et al., 1996; Asif et al., 2000; Vaitkevicius et al., 2001; Aronson, 2003; Candido et al., 2003; Liu et al., 2003; Bakris et al., 2004; van Heerebeek et al., 2008). However, its role in the development of diastolic dysfunction secondary to impaired ventricular relaxation, determined principally by the rate of resequestration of $\mathrm{Ca}^{2+}$ into the SR rather than by an increased myocardial fibrosis, is not known. In the present study, we investigated a novel mechanism by which AGE accumulation functionally impairs SR $\mathrm{Ca}^{2+}$ regulatory proteins (especially SERCA pump), by use of an antiglycation therapeutic agent: dimethyl-3-phenacylthiazolium chloride (alagebrium chloride or ALT-711), which chemically breaks AGE cross-links. This compound has been tested in several pre-clinical animal studies, and has been shown to significantly reduce cardiac AGE level in STZ-induced diabetic rats and prevent diabetesinduced structural changes in the myocardium (Asif et al., 2000; Vaitkevicius et al., 2001; Candido et al., 2003; Liu et al., 2003; Vasan et al., 2003; Bakris et al., 2004; Cooper, 2004). In contrast with inhibitors of AGE cross-link (e.g., aminoguanidine), AGE cross linkage breakers prevent but also reverse the crosslink process once it has already been established (Norton et al., 1996). Therefore, one could argue that similar beneficial therapeutic effects of ALT-711, as the ones observed in this study, could be obtained once diabetic cardiomyopathy has been established. Since ALT-711 therapy was administered at the onset of diabetes in our study, further studies will be required to confirm its therapeutic effect in subjects with established diabetes. Overall, our in vivo data suggested that long-term treatment with ALT-711 improved the clinical condition of treated diabetic rats, as evident by the increase (although not statistically significant) in body weight. Importantly, ALT-711 therapy partially improved diastolic function, as evident by the attenuation of the prolongation in isovolumic relaxation time and MPI observed in treated 


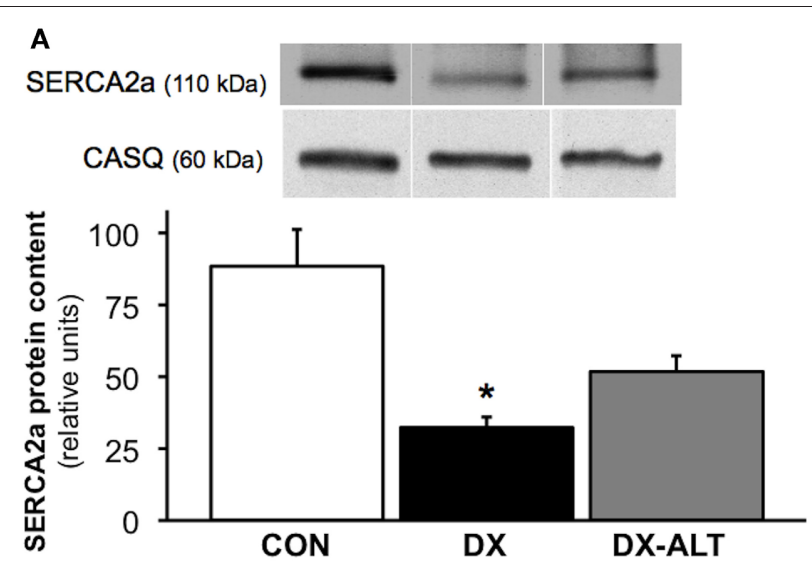

B

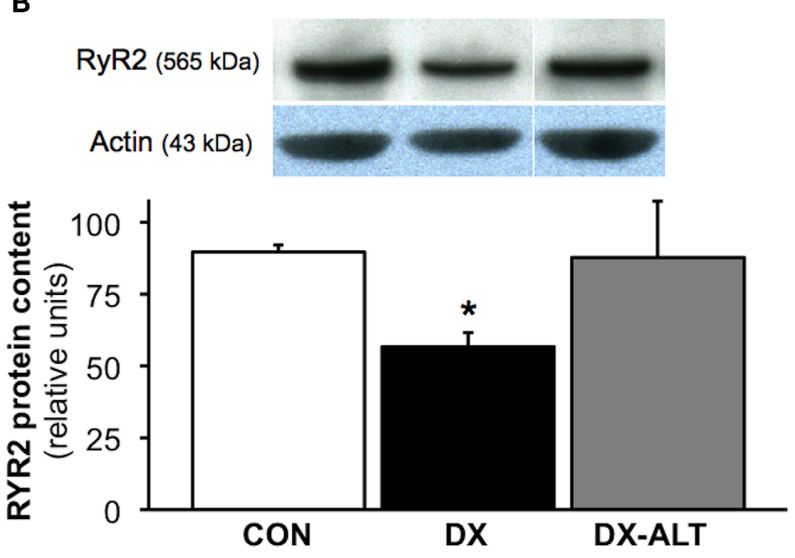

FIGURE 6 | ALT-711 therapy partially attenuated the decreased expression of $\mathrm{SR} \mathrm{Ca}^{2+}$ regulatory proteins in the diabetic myocardium. (A) Top panel: representative immunoblot of sarco(endo)plasmic reticulum $\mathrm{Ca}^{2+}$-ATPase (SERCA2a) and calsequestrin (loading control) expression in the myocardium of control (CON), untreated diabetic (DX), treated diabetic (DX-ALT) groups; samples were from the same membrane, which was reprobed for calsequestrin (the loading control). Within the same gel, the reassembly of noncontiguous lanes has been demarcated by white spaces. Bottom panel: normalized optical density (OD; relative to calsequestrin) of SERCA2a protein content was significantly decreased in DX, but not in DX-ALT, hearts compared to control group. Data are mean \pm SE for $n=4-5 /$ group. (B) Top panel: representative immunoblot of Ryanodine Receptor (RyR2) protein expression in the myocardium of control (CON) untreated diabetic (DX), treated diabetic (DX-ALT) hearts (samples were from the same membrane, which was reprobed for actin, the loading control. Within the same gel, the reassembly of noncontiguous lanes has been demarcated by white spaces). Bottom panel: normalized optical density (relative to actin, loading control) of RyR2 protein was significantly decreased in DX, but unchanged in DX-ALT, hearts compared to control group. Data are mean \pm SE for $n=5$ /group. ${ }^{*} P<0.05$ when comparing values from age-matched control heart. content, which are all consistent with decreased $\mathrm{SR} \mathrm{Ca}^{2+}$ reuptake during the relaxation phase, as previously reported by our group (Lacombe et al., 2007). Extensive studies have tried to unravel metabolic disturbances and intracellular targets that lead to impaired $\mathrm{Ca}^{2+}$ homeostasis and to the diabetic cardiomyopathic phenotype, a complex multifactorial disorder (Poornima et al., 2006). AGE accumulation during diabetes, and to a less extent during aging, could contribute to the observed cardiomyopathy, since AGEs form irreversible cross-links with many proteins with low turnover rates, such as collagen but also intracellular cardiac SR proteins (i.e., SERCA2a pump and RyR2); however, their pathogenic role on excitation-contraction coupling has not been investigated. Since treatment with AGE crosslink breakers has been shown to completely prevent or reduce the formation of AGEs (Wolffenbuttel et al., 1998; Cooper et al., 2000; Vaitkevicius et al., 2001; Candido et al., 2003; Vasan et al., 2003; Forbes et al., 2004), ALT-711 treatment could have a potential beneficial effect on the function of $\mathrm{SR} \mathrm{Ca}^{2+}$ regulatory proteins. Following ALT-711 therapy, we observed a normalization of the $\mathrm{Ca}^{2+}$ transient decay, and a partial restoration of intra-SR $\mathrm{Ca}^{2+}$ stores and SERCA2a protein expression in diabetic cardiac myocytes. These data suggested that ALT-711 treatment decreased excess accumulation of AGEs on the SERCA pump by breaking the cross-links that form during diabetic cardiomyopathy, resulting in partial improvement of SERCA activity and SR $\mathrm{Ca}^{2+}$ reuptake. The enhanced SR $\mathrm{Ca}^{2+}$ reuptake during the relaxation phase of cardiac myocytes resulted in partial improvement of in vivo diastolic function in treated diabetic subjects.

In contrast, treatment with the AGE cross-link breaker for 8 weeks did not normalize $\mathrm{Ca}^{2+}$ transient amplitude in isolated diabetic myocytes. In correlation with these in vitro findings, we observed a moderate, but persistent, reduction of cardiac contractility in diabetic animals treated with ALT-711, as evident by the mild decrease in EF in both untreated and treated diabetic animals. One surprising finding was the persistent decrease in $\mathrm{Ca}^{2+}$ transient amplitude in the face of partially restored $\mathrm{SR} \mathrm{Ca}^{2+}$ load and RyR2 protein expression in the diabetic myocardium after ALT-711 treatment. Since abnormal SR Ca ${ }^{2+}$ release during diastole has been reported in diabetic myocytes (Shao et al., 2007), one could hypothesize that ALT-711 therapy may also improve $\mathrm{Ca}^{2+}$ homeostasis by stabilizing RyR mediated $\mathrm{SR} \mathrm{Ca}^{2+}$ release during the relaxation phase of diabetic cardiac myocytes and that AGE accumulation may also impair RyR function leading to diastolic SR $\mathrm{Ca}^{2+}$ leak. Therefore, these data further support the concept that AGE accumulation may play a larger pathogenic role during diastolic dysfunction and diastolic heart failure than during systolic dysfunction (Hartog et al., 2007).

\section{CONCLUSIONS AND CLINICAL SIGNIFICANCE}

Treatment with an AGE cross-link breaker partially attenuated the alterations associated with cardiac function and SR $\mathrm{Ca}^{2+}$ handling during diabetic cardiomyopathy. Since diabetic cardiomyopathy is a multifactorial disorder, these data suggest that AGE accumulation contributes to the impairment in excitation-contraction coupling by altering the function of SR 
$\mathrm{Ca}^{2+}$ regulatory proteins, leading to a decreased ability for the diabetic myocardium to relax. Therefore, findings from this study provide novel mechanistic insights related to the pathogenic role of AGE accumulation on SR $\mathrm{Ca}^{2+}$ handling in cardiac myocytes. Finally, since there is currently a lack of specific therapy to improve LV relaxation, findings from this study could have direct practical implications for the development of therapeutic strategies for patients with diabetic cardiomyopathy.

\section{REFERENCES}

Amos, A. F., McCarty, D. J., and Zimmet, P. (1997). The rising global burden of diabetes and its complications: estimates and projections to the year 2010. Diabet. Med. 14, S1-S85.

Aronson, D. (2003). Cross-linking of glycated collagen in the pathogenesis of arterial and myocardial stiffening of aging and diabetes. $J$. Hypertens. 21, 3-12.

Asif, M., Egan, J., Vasan, S., Jyothirmayi, G. N., Masurekar, M. R., Lopez, S., Williams, C., Torres, R. L., Wagle, D., Ulrich, P., Cerami, A., Brines, M., and Regan, T. J. (2000). An advanced glycation end product cross-link breaker can reverse age-related increases in myocardial stiffness. Proc. Natl. Acad. Sci. U.S.A. 97, 2809-2813.

Aurigemma, G. P., Zile, M. C., and Gaasch, W. H. (2006). Contractile behavior of the left ventricle in diastolic heart failure: with emphasis on regional systolic function. Circulation 113, 296-304.

Bakris, G. L., Bank, A. J., Kass, D. A., Neutel, J. M., Preston, R. A., and Oparil, S. (2004). Advanced glycation end-product cross-link breakers. A novel approach to cardiovascular pathologies related to the aging process. Am. J. Hypertens. 17, 23S-30S.

Bers, D. M. (2002). Cardiac excitationcontraction coupling. Nature 415, 198-205.

Bidasee, K. R., Nallani, K., Yu, Y., Cocklin, R. R., Zhang, Y., Wang, M., Dincer, U. D., Besch, H. R. Jr. (2003). Chronic diabetes increases advanced glycation end products on cardiac ryanodine receptors/calcium-release channels. Diabetes 52, 1825-1836.

Bidasee, K. R., Zhang, Y., Shao, C. H., Wang, M., Patel, K. P., Dincer, U. D., and Besch, H. R. Jr. (2004). Diabetes increases formation of advanced glycation end products on Sarco(endo)plasmic reticulum $\mathrm{Ca}^{2+}$-ATPase. Diabetes 53, 463-473.

Boudina, S., and Abel, E. D. (2007). Diabetic cardiomyopathy revisited. Circulation 115, 3213-3223.
Candido, R., Forbes, J. M., Thomas, M. C., Thallas, V., Dean, R. G., Burns, W. C., Tikellis, C., Ritchie, R. H., Twigg, S. M., Cooper, M. E., and Burrell, L. M. (2003). A breaker of advanced glycation end products attenuates diabetes-induced myocardial structural changes. Circ. Res. 92, 785-792.

Choi, K. M., Zhong, Y., Hoit, B. D., Grupp, I. L., Hahn, H., Dilly, K. W., Guatimosim, S., Lederer, W. J., and Matlib, M. A. (2002). Defective intracellular $\mathrm{Ca}^{2+}$ signaling contributes to cardiomyopathy in Type 1 diabetic rats. Am. J. Physiol. Heart Circ. Physiol. 283, H1398-H1408.

Cooper, M. E. (2004). Importance of advanced glycation end products in diabetes-associated cardiovascular and renal disease. Am. J. Hypertens. 17, 31S-38S.

Cooper, M. E., Thallas, V., Forbes, J., Scalbert, E., Sastra, S., Darby, I., and Soulis, T. (2000). The cross-link breaker, N-phenacylthiazolium bromide, prevents vascular advanced glycation end-product accumulation. Diabetologia 43, 660-664.

Dent, C. L., Bowman, A. W., Scott, M. J., Allen, J. S., Lisauskas, J. B., Janif, M., Wickline, S. A., and Kovacs, S. J. (2001). Echocardiographic characterization of fundamental mechanisms of abnormal diastolic filling in diabetic rats with a parameterized diastolic filling formalism. J. Am. Soc. Echocardiogr. 14, 1166-1172.

Dirksen, W. P., Lacombe, V. A., Chi, M., Kalyanasundaram, A., ViatchenkoKarpinski, S., Terentyev, D., Zhou, Z., Vedamoorthyrao, S., Li, N., Chiamvimonvat, N., Carnes, C. A. Franzini-Armstrong, C., Györke, S., and Periasamy, M. (2007). A mutation in calsequestrin CSQD307H impairs SR calcium storage and release functions and causes polymorphic ventricular tachycardia in mice. Cardiovasc. Res. 75, 69-78.

Fang, Z. Y., Prins, J. B., and Marwick, T. H. (2004). Diabetic cardiomyopathy: evidence, mechanisms, and therapeutic implications. Endocr. Rev. 25, 543-567.

Forbes, J. M., Yee, L. T., Thallas, V., Lassila, M., Candido, R.,

\section{ACKNOWLEDGMENTS}

We gratefully acknowledge Natalie Virell, Hsiang-Ting Ho, Dr. Amanda Waller and Dr. Amy Gerwitz for their excellent technical assistance with the experiments and data analysis. Support was provided by the American Heart Association, Great River affiliate (Grant-In-Aid to Véronique A. Lacombe) and by National Institutes of Health Grants (to Véronique A. Lacombe and R00 HL091056 to Brandon J. Biesiadecki).

Jandeleit-Dahm, K. A., Thomas, M. C., Burns, W. C., Deemer, E. K., Thorpe, S. M., Cooper, M. E., and Allen, T. J. (2004). Advanced glycation end product interventions reduce diabetesaccelerated atherosclerosis. Diabetes 53, 1813-1823.

Hartog, J. W., Voors, A. A., Bakker, S. J., Smit, A. J., and van Veldhuisen, D. J. (2007). Advanced glycation end-products (AGEs) and heart failure: pathophysiology and clinical implications. Eur. J. Heart Fail. 9, 1146-1155.

Kubalova, Z., Terentyev, D. Viatchenko-Karpinski, S., Nishijima, Y., Györke, I., Terentyeva, R., da Cunha, D. N., Sridhar, A., Feldman, D. S., Hamlin, R. L., Carnes, C. A., and Györke, S. (2005). Abnormal intrastore calcium signaling in chronic heart failure. Proc. Natl. Acad. Sci. U.S.A. 102, 14104-14109.

Lacombe, V. A., Terentyev, D., Viatchenko-Karpinski, S., Hamlin, R. L., Györke, S., and Carnes, C. (2010). Diltiazem treatment attenuates arrhythmogenesis during diabetic cardiomyopathy by stabilizing ryanodine receptors-mediated sarcoplasmic reticulum calcium release (abstract). Circulation 122, A20958.

Lacombe, V. A., Viatchenko-Karpinski, S., Terentyev, D., Sridhar, A., Emani, S., Bonagura, J. D., Feldman, D. S., Györke, S., and Carnes, C. A. (2007). Mechanisms of impaired calcium handling underlying subclinical diastolic dysfunction in diabetes. Am. J. Physiol. Regul. Integr. Comp. Physiol. 293, R1787-R1797.

Lagadic-Gossmann, D., Buckler, K. J., Le Prigent, K., and Feuvray, D. (1996). Altered $\mathrm{Ca}^{2+}$ handling in ventricular myocytes isolated from diabetic rats. Am. J. Physiol. 270, H1529-H1537.

Liu, J., Masurekar, M. R., Vatner, D. E., Jyothirmayi, G. N., Regan, T. J., Vatner, S. F., Meggs, L. G., and Malhotra, A. (2003). Glycation endproduct cross-link breaker reduces collagen and improves cardiac function in aging diabetic heart. Am.
J. Physiol. Heart Circ. Physiol. 285, H2587-H2591.

Ma, H., Li, S. Y., Xu, P., Babcock, S. A., Dolence, E. K., Brownlee, M., Li, J., and Ren, J. (2009). Advanced glycation endproduct (AGE) accumulation and AGE receptor (RAGE) up-regulation contribute to the onset of diabetic cardiomyopathy. J. Cell. Mol. Med. 13, 1751-1764.

Meurs, K., Lacombe, V. A., Dryburgh, K., Fox, P. R., and Kittleson, M. D. (2006). Differential expression of the cardiac ryanodine protein in normal dogs and boxer dogs with arrhythmogenic right ventricular cardiomyopathy. Hum. Genet. 120, 111-118.

Netticadan, T., Temsah, R. M., Kent, A., Elimban, V., and Dhalla, N. S. (2001). Depressed levels of $\mathrm{Ca}^{2+}$. cycling proteins may underlie sarcoplasmic reticulum dysfunction in the diabetic heart. Diabetes 50, 2133-2138.

Norton, G. R., Candy, G., and Woodiwiss, A. J. (1996). Aminoguanidine prevents the decreased myocardial compliance produced by streptozotocininduced diabetes mellitus in rats. Circulation 93, 1905-1912.

Piccini, J. P., Klein, L., Gheorghiade, M., and Bonow, R. O. (2004). New insights into diastolic heart failure: role of diabetes mellitus. Am. J. Med. $116,64 S-75 S$.

Pierce, G. N., and Russell, J. C. (1997). Regulation of intracellular $\mathrm{Ca}^{2+}$ in the heart during diabetes. Cardiovasc. Res. 34, 41-47.

Poornima, I., Parikh, P., and Shannon, R. (2006). Diabetic cardiomyopathy: the search for a unifying hypothesis. Circ. Res. 98, 596-605.

Ratnadeep, B., Gavin, O. Y., Xiuhua, W., Liyan, Z., John, U. R., Gary, L. D., and Zamaneh, K. (2009). Type 1 diabetic cardiomyopathy in Akita (Ins2 WT/C96Y) mouse model is characterized by lipotoxicity and diastolic dysfunction with preserved systolic function. Am. J. Physiol. Heart Circ. Physiol. 297, H2096-H2108. 
Shao, C. H., Rozanski, G. J., Patel, K. P., and Bidasee, K. R. (2007). Dyssynchronous (non-uniform) $\mathrm{Ca}^{2+}$ release in myocytes from streptozotocin-induced diabetic rats. J. Mol. Cell. Cardiol. 42, 234-246.

Su, H. M., Lin, T. H., Voon, W. C., Lee, K. T., Chu, C. S., Lai, W. T., and Sheu, S. H. (2006). Differentiation of left ventricular diastolic dysfunction, identification of pseudonormal/restrictive mitral inflow pattern and determination of left ventricular filling pressure by Tei index obtained from tissue Doppler echocardiography. Echocardiography 23, 287-294.

Vaitkevicius, P. V., Lane, M., Spurgeon, H., Ingram, D. K., Roth, G. S., Egan, J. J., Vasan, S., Wagle, D. R., Ulrich, P., Brines, M., Wuerth, J. P., Cerami, A., and Lakatta, E. G. (2001). A cross-link breaker has sustained effects on arterial and ventricular properties in older rhesus monkeys. Proc. Natl. Acad. Sci. U.S.A. 98, 1171-1175.

van Heerebeek, L., Hamdani, N., Handoko, M. L., Falcao-Pires, I., Musters, R. J., Kupreishvili, K., Ijsselmuiden, A. J., Schalkwijk, C. G., Bronzwaer, J. G., Diamant, M., Borbély, A., van der Velden, J., Stienen, G. J., Laarman, G. J., Niessen, H. W., and Paulus, W. J. (2008). Diastolic stiffness of the failing diabetic heart. Importance of fibrosis, advanced glycation end products, and myocyte resting tension. Circulation 117, 43-51.

Vasan, S., Foiles, P., and Founds, H. (2003). Therapeutic potential of breakers of advanced glycation end product-protein crosslinks. Arch. Biochem. Biophys. 419, 89-96.

Ware, B., Bevier, M., Nishijima, Y., Rogers, S., Carnes, C. A., and Lacombe, V. A. (2011). Chronic heart failure selectively induces regional heterogeneity of insulinresponsive glucose transporters.
Am. J. Physiol. Regul. Integr. Comp. Physiol. 301, R1300-R1306.

Wolffenbuttel, B. H., Boulanger, C. M., Crijns, F. R., Huijberts, M. S., Poitevin, P., Swennen, G. N., Vasan, S., Egan, J. J., Ulrich, P., Cerami, A., and Levy, B. I. (1998). Breakers of advanced glycation end products restore large artery properties in experimental diabetes. Proc. Natl. Acad. Sci. U.S.A. 95, 4630-4634.

Zhong, Y., Ahmed, S., Grupp, I. L., and Matlib, M. A. (2001). Altered SR protein expression associated with contractile dysfunction in diabetic rat hearts. Am. J. Physiol. Heart Circ. Physiol. 281, H1137-H1147.

Conflict of Interest Statement: The authors declare that the research was conducted in the absence of any commercial or financial relationships that could be construed as a potential conflict of interest.
Received: 11 May 2012; paper pending published: 02 June 2012; accepted: 04 July 2012; published online: 19 July 2012. Citation: Kranstuber AL, del Rio C, Biesiadecki BJ, Hamlin RL, Ottobre J, Gyorke S and Lacombe VA (2012) Advanced glycation end product crosslink breaker attenuates diabetes-induced cardiac dysfunction by improving sarcoplasmic reticulum calcium handling. Front. Physio. 3:292. doi: 10.3389/fphys. 2012.00292

This article was submitted to Frontiers in Integrative Physiology, a specialty of Frontiers in Physiology.

Copyright (c) 2012 Kranstuber, del Rio, Biesiadecki, Hamlin, Ottobre, Gyorke and Lacombe. This is an open-access article distributed under the terms of the Creative Commons Attribution License, which permits use, distribution and reproduction in other forums, provided the original authors and source are credited and subject to any copyright notices concerning any third-party graphics etc. 